\title{
Neuroprotection and visual function after optic neuritis
}

Axel Petzold*

September 19, 2016

*The Dutch Expertise Center for Neuro-ophthalmology, VU University Medical Center, Amsterdam, De Boelelaan 1117, 1081 HV Amsterdam, NL. Email: a.petzold@vumc.nl

\section{Abstract}

Purpose of review: To discuss the advantages and pitfalls of testing neuroprotective treatment strategies in patients suffering from optic neuritis.

Recent findings: Spectral domain optical coherence tomography (OCT) now permits for automated segmentation of individual retinal layers. The peripapillary retinal nerve fibre layer (pRNFL) has been used in 13 of the 15 trials reviewed. Twelve trials made use of electrophysiology. Crucially, the overestimation of good visual recovery has been recognised. Assessment of low contrast visual acuity and colour vision are now mainstream.

Summary: Advantages are the availability of highly accurate and robust trial outcome measures. As a single long-term structural outcome measure the pRNFL is sufficient. For shorter term assessment the GCIPL and axonal birefringence are promising. Longitudinal blood levels of neurofilament proteins permit to recognise axonal loss at presentation and monitor changes longitudinally. Inner nuclear layer (INL) volume changes relate to inflammatory disease activity.

Pitfalls are related to the timing of events. Hyperacute recruitment is needed for future trials. The onset of demyelination is not known which complicates timing of electrophysiological recordings. Optic disc oedema precludes the use of the pRNFL from the affected eye as a baseline variable. The concomitant use of corticosteroids complicates interpretation of trial data.

Keywords:

\section{Contents}

1 Introduction

2 Optic neuritis in multiple sclerosis

3 Relevant trial design implications from the diagnostic work-up of acute optic neuritis

4 Outcome measures for neuroprotective treatment trials in optic neuritis

4.1 Electrophysiology

4.2 $\underline{\text { OCT }}$

5 Pupillography

5.1 MRI

6 Neuroprotective treatment trials

7 Conclusion

8 Acknowledgement

$\underline{\text { References }}$

\section{Introduction}

Optic neuritis $(\mathrm{ON})$ has been rediscovered as a model disease for human treatment trials in demyelinating diseases [1-3]. With few exceptions the multiple sclerosis (MS) treatment trial community had turned a blind eye on MS associated ON (MSON) since completion of the influential Optic Neuritis Treatment Trial (ONTT). What has triggered the renewed interest in $\mathrm{ON}$ for human treatment trials?

Treatment of inflammatory disease activity MS is effective. Disease modifying treatments (DMT) reduce the chance for a new relapse by about $30-70 \%$ depending on choice of treatment [4]. Evidence of DMT to halt disease progression has been less convincing.

The attraction of $\mathrm{ON}$ as a model disease for neuroprotective treatment trials coincided with the availability of new clinical and paraclinical outcome measures for vision [5]. The expectations are that a treatment trial in MSON can be conducted fast with highly accurate outcome measures. A successful MSON trial can be a promising stepping stone for neuroprotective treatment strategies in MS at large.

\section{Optic neuritis in multiple sclerosis}

The presentation of MSON is that of peri-/retro-orbital pain worsening with eye movements which is associated with loss of vision [6]. Recovery is ususally good. Residual visual symptoms are dyschromatopsia and reduced low contrast visual acuity $[5,6]$. Some patients have difficulties with perception of movements in depth (Pulfrich phenomenon) and transient worsening of vision related to changes in body temperature (Uhthoff phenomenon).

\section{Relevant trial design implications from the diagnostic work-up of acute optic neuritis}

Ahmet Toosy has reviewed the general assessment of patients with MSON in this issue of the Journal. Practically, the diagnosis of acute ON within days after onset is much more challenging than after about three months [6]. Many of the classical symptoms and signs will not yet have developed. Recognising the about 5\% of patients with ON who have a poor visual outcome at presentation is challenging [7]. Contamination of neuroprotective optic neuritis treatment trials with these patients is a real possibility. Exclusion of other aetiologies of autoimmune visual loss in the acute phase requires testing for auto-antibodies $[8,9]$.

\section{Outcome measures for neuroprotective treatment trials in optic neuritis}

The key pathological features used as an outcome measure in MSON are demyelination and axonal loss. Currently, demyelination is recorded using electrophysiology. Axonal loss in the retina is assessed with OCT. Axonal loss in the optic nerve, optic tracts and optic radiations is assessed by MRI.

Visual function is assessed with high and low contrast visual acuities, contrast sensitivity, colour vision tests and visual field tests [5, 6].

Visual quality of live is documented with validated patient questionnaires. The 25-Item National Eye Institute Visual Function Questionnaire (NEI-VFQ-25) has been extended by a neuro-ophthalmology supplement [10].

Remyelination Inflammation at onset of MSON is sufficient to cause conduction block in the optic nerve. Inflammation precedes demyelination. Because the nadir of demyelination is not known, assessment of demyelination/remyelination is difficult. It is not known at which time point after onset of acute MSON visual function and electrophysiology are best to be recorded. This will remain a tricky problem for optic neuritis treatment trial design. 
The electrophysiological tests are of particular value in studies evaluating therapy [3, 12]. The International Society for Clinical Electrophysiology of Vision (ISCEV) (www.iscev.org/standards/proceduresguide.html) specifies the standards, gives recommendations and provides guidelines on testing [13]. For optic neuritis treatment trials relevant tests to consider are the Pattern Appearance Visually Evoked Cortical Potential (VEP) and the Pattern Electroretinogram (PERG) [6, 14]. Demyelination of the optic pathways results in delay of the VEP P100 amplitude. The accepted wisdom is that recovery of conduction velocity indicates remyelination. The challenge is to capture the right moment.

The PERG not only permit for early detection of retinal pathology, but also shows a reduction of the N95 component in affected eyes [15]. The multifocal ERG (mfERG) permits for more detailed spacial analysis and shows a reduced optic nerve head component (ONHC) response following demyelination [16].

\subsection{OCT}

Retinal has become one of the best validated and sensitive outcome measures for neurodegeneration following MSON [5, 6, 17]. The field recognised early the need for standardised OCT acquisition procedures, quality control (QC) and reporting guidelines [18-20].

RNFL The peripapillary retinal nerve fibre layer (pRNFL)is a higly robust long term outcome measure for MSON treatement trials. Following optic neuritis there is substantial axonal damage [7]. At presentation this can be masked by presence of optic disc oedema [21]. Longitudinal studies demonstrate that atrophy of the pRNFL becomes manifest within about three months and plateaus after about one year [2, 22]. Atrophy of the pRNFL after MSON in a meta-analysis of cross-sectional studies averaged at $20 \mu \mathrm{m}(95 \% \mathrm{CI} 18-23 \mu \mathrm{m})$ using time domain OCT [23]. The annual atrophy rate of the pRNFL was highest in patients with MSON and a short disease duration $[24,25]$.

GCIPL The ganglion cell / inner plexiform layer (GCIPL) is a valuable early response outcome measure. The GCIPL has the important advantage that optic disc oedema does not reach the macular, hence atrophy can be detected about two month earlier than in the RNFL [22].

INL The inner nuclear layer (INL) is a promissing outcome measure for inflammation. In MS there is evidence that INL volume changes are related to inflammatory disease activity and respond to DMT [26]. These rapid transient volume changes were hypothesised to be caused by fluid shift in an impairment retinal glymphatic system [27]. Longitudinal INL volume assessments may inform on inflammatory disease activity in MSON treatment trials.

\section{Pupillography}

As discussed in detail by Finn Bremner in this issue, infrared video pupillography permits with high accuracy to record of a dynamic pupil response under controlled conditions. This provides an objective assessment of the relative afferent pupillary defect (RAPD) observed in MSON. The development of hand held pupillometer devices has now reached a level of reliability suitable for multi-centre trials [28].

\subsection{MRI}

Optic neuritis treatment trials may include MRI for diagnostic reasons, to exclude other relevant co-morbidity and for safety reasons. The recommended sequences for the optic nerve are to be taken in coronal orientation and take about 10 minutes. They include T2-weighted images with fat suppression (e.g., STIR, SPIR) and T1-weighed images before and after contrast administration $[6,29,30]$. For assessment of tissue integrity a range of additional techniques are available, such as high resolution $3 \mathrm{D}$ T2weighted (CISS) images for the visualisation and volume measurements of the optic nerve and diffusion tensor imaging (DTI) [17, 31]. There is experimental and human post-mortem evidence to suggest a role of DTI and high field MRI for assessment of remyelination [32]. Longitudinal studies will need to take into account that there is a relevant effect of using different scanners over time [33].

\section{Neuroprotective treatment trials}

Figure 1 gives an overview on compounds and strategies reviewed. The primary and secondary outcome measures have been summarised in Table 1 .

Adrenocorticotropic hormone One phase 0 (NCT01987167) and one phase 4 (NCT01838174) investigate the neuroprotective role of adrenocorticotropic hormone (ACTH, synonymous corticotropin in MSON. It is hypothesised that melanocortin-mediated anti-inflammatory effects of ACTH will be neuroprotective in MSON. Patients in one trial (NCT01838174) also receive intravenous methylprednisolone therapy (IVMP).

Amiloride Two phase 2 trials (NCT01879527, NCT01802489) test for the neuroprotective effect of amiloride in MSON [34]. Amiloride is approved as a diuretic. Amiloride blocks the acid sensing ion channel 1 (ASIC1) which controls $\mathrm{Na} 2+$ and $\mathrm{Ca} 2+$ fluxes. Blockage of cytotoxic cellular overload with Ca2+ could be neuroprotective in MSON. The primary outcome measure was the scanning laser polarimetry (GDx) derived pRNFL in addition to the OCT pRNFL. The GDx methodology relies on birefringence of the RNFL [23]. Birefringence is related to the structure of dominant axonal filaments such as the neurofilament protein. Because neurofilament protein composition changes with axonal injury and precedes axonotmesis it had been hypothesised that changes of RNFL birefringence may represent the earliest sign of axonal degeneration in MSON [23]. The Amiloride trial in MSON has however been reported as negative with regard to the primary outcome measures (ECTRIMS, 15.09.2016, McKee et al., Talk 102). What can be learned from the trial however, is that the baseline GDX pRNFL was less affected by optic disc oedema compared to the OCT derived pRNFL.

Unexpectedly one of the secondary outcome measures, the VEP latencies, were found to be delayed by $12.81 \mathrm{~ms}$ in the treatment arm (27.27 ms) if compared to placebo $(14.45, \mathrm{p}=0.0043)$ at six months. This may imply that amiloride impedes remyelination.

It should also be remembered that patients were recruited within up to 28 days (mean 14 days) of onset which may have been too late.

Atacicept One phase 2 trial (2007-003925-26) gave the recombinant fusion protein atacicept. Atacicept inhibits B-cells. Mature B- and plasma cells are more likely to blocked then memory B-cells and progenitor cells. Corticosteroids may be given optionally to treat the initial ON event. The trial was prematurely stopped. Disease activity was higher in the treatment arm [35]. The data available showed a minimal effect of atacicept on pRNFL atrophy.

Cholecalciferol There is evidence patients with MSON and low vitamin D levels have more severe atrophy of the pRNFL and GCL [36]. There is one ongoing phase 3 (2010-018498-39) testing the potential neuroprotective effect of cholecalciferol in acute MSON.

Clemastine An exciting translational research line as lead to the discovery of clemastine fumarate as a compound stimulation remyelination [37]. Clemastine (meclastin) has antihistaminic and anticholinergic properties and is used for treatment of allergic rhinitis. A phase 2 study (NCT02040298) demonstrated significant improvement of P100 latency delay by about 1.9 ms/eye during the treatment period (presented at the American Academy of Neurology's 68th Annual Meeting in Vancouver, Canada, April 15 to 21, 2016). A phase 2 trial for acute MSON is ongoing (NCT02521311). Patients will also receive IVMP or oral prenisolone.

Corticosteroids One major result of the ONTT was that most neurologists switched from oral to intravenous corticosteroid treatment for MSON [38]. An important recent trial demonstrated the non inferiority of oral high-dose (1000 mg) methylprednisolone for 3 days compared to IVMP (1000 mg) [39]. No placebo controlled trial of oral corticosteroids had been published since 1999 according to the last systematic Chochrane review [40]. Since, a randomised single blinded clinical trial had completed suggesting no inferiority of oral compared to intravenous corticosteroids (ECTRIMS 15.09.2016, S.A. Morron, poster 660).

There is anecdotal evidence that hyperacute treatment with corticosteroids in MSON may be neuroprotective [41, 42]. One phase 4 trial (2012-002628-34) evaluates early treatment with high-dose IVMP.

Erythropoietin The glycoprotein hormone erythropoietin (EPO) boosts red blood cell production. Consistent with experimental evidence for neuroprotective properties of 
EPO a phase 2 MSON treatment trial (NCT00355095) was successfully concluded [43]. Treatment with EPO lead to significantly less pRNFL atrophy (OCT), optic nerve atrophy (MRI) and better VEP latencies. Patients in this trial also received IVMP. The median time from onset to treatment was 4 days for EPO/IVMP and two days later for IVMP. Whether or not early treatment with IVMP may have caused a bias is not known. A follow-up phase 3 trial (NCT01962571) is ongoing [44].

Fingolimod A phase 2/3 trial with Fingolimod (2011-004787-30) in MSON was prematurely terminated due to poor recruitement.

Minocycline Two phase 2 trials (NCT01073813, 2011-001589-16) examine the neuroprotective effect of the tetracycline antibiotic minocycline in MSON. Minocycline has multi-level anti-apoptotic properties and reduces glutamate toxicity.

Opicinumab Differentiation of oligodendrocytes is inhibited by membrane glycoproteins to which LINGO-1 belongs [3]. A phase 2 trial with Opicinumab (NCT01721161) has completed. Treatment with Opicinumab was associated with an increased VEP latency recovery $(22.24 \mathrm{~ms})$ compared to placebo (14.69, $\mathrm{p}=0.0504)$. There was no effect on the pRNFL $(-11.75 \mu \mathrm{m}$ vs $15.66 \mu \mathrm{m})$, GCIPL (-9.9 vs -11.05$)$ or LCVA. The trial has been extended (NCT02657915).

Phenytoin There is good translational and preclinical data on the neuroprotective effect of ion channel blocking agents [34]. A phase 2 trial with the anti-epileptic drug phenytoin was positive for the primary outcome measure. There was significantly less atrophy $(-7.15 \mu \mathrm{m})$ of the pRNFL in the treatment group if compared to the placebo group [45]. Likewise thinning of the total macular volume $\left(-0.20 \mathrm{~mm}^{3}\right)$ was less marked in the treatment group. Atrophy of the GCIPL, thought to be a good biomarker because less affected by the baseline disc oedema compared to the pRNFL, did however not differ significantly between the treatment group and placebo (ECTRIMS, 15.09.2016, R. Kapoor, Talk 99). Of the secondary outcome measures, blood derived neurofilament heavy chain levels were significantly lower in patients treated with phenytoin compared to placebo (ECTRIMS 16.09.2016, S. Gnanapavan et al. Poster 1619). None of the clinical outcome measures reached significance.

The average time from onset to randomisation was 8 days in both groups. Patients were given the choice to have IVMP. More patients in the treatment group received IVMP (85\%) compared to the placebo group (75\%).

Simvastatin There is in-vitro, translational, preclinical and clinical evidence for favourable immunological effects and neuroprotection of statins in demyelination, particularly in secondary progressive MS [46]. A phase 3 trial suggested improved recovery of colour vision and VEP latency amplitude in patients treated with statins [47]. The average time from symptom onset to treatment was 12 days for statins and 15 days for placebo.

\section{Conclusion}

Compared to the past two decades, the last two years have been exiting and thought stimulating data regarding the treatment of patients with MSON. Six compounds are in phase 2 and 5 in phase 3/4. With one exception, opicinumab, all compounds have already been approved for different conditions.

Retinal OCT has clearly become the favourable structural outcome measure, being included in $13 / 15(86 \%)$ of the trials. The most frequently employed functional paraclinical outcome measure is the VEP latency in 12/15 (80\%) of trials. Well thought through timing of the VEP assessement is crucial to capture the moment of optic nerve demyeliation. Presently, short interval longitudinal VEP recordings may be required.

Because of the small effect size of the pRNFL and VEP latencies in the published trials it cannot be overemphasised how important it is to have a rigorous quality control pipeline in place in order to avoid data contamination [Sch2014_c, 13, 19, 20].

Ten studies detailed MRI sequences with assessment of optic nerve diameter being useful for assessment of atrophy. Likewise coronal T2 weighted images with fat suppression and coronal T1 Gd enhancing images are extremely useful for confirmation of presence and location of optic nerve inflammation. There is a plethora of exciting MRI research sequences which investigate diffuse tissue damage.

Of the novel outcome measures two trials included a biomarker for axonal degeneration, the neurofilament protein; one trial employed pupillography.

The time from symptom onset to treatment initiation in MSON is still rather long compared to what has been achieved in stroke medicine. There is anecdotal evidence for a beneficial effect of hyperacute treatment. We have achieved same day treatment in the vast majority of patients presenting with transient monocular blindness at Moorfields Eye Hospital by tapping into established stroke pathways. Similar efforts need to be done for MSON.

\section{Acknowledgement}

My thanks go to Professor Graham Holder and his insightful comments on the challenges ahead for optimal timing of VEP assessment to capture demyelination (and remyelination) in the optic nerve.

\section{References}

$[1]$

J. L. Bennett, M. Nickerson, F. Costello, et al. "Re-evaluating the treatment of acute optic neuritis.” J Neurol Neurosurg Psychiatry 86 (2015), pp. $799-808$.

$[2]$

F. Costello, Y. I. Pan, E. A. Yeh, et al. "The temporal evolution of structural and functional measures after acute optic neuritis." Journal of Neurology, Neurosurgery \& Psychiatry (2015), pp. 1369-1373.

[3]

O. Aktas, P. Albrecht, and H.-P. Hartung. "Optic neuritis as a phase 2 paradigm for neuroprotection therapies of multiple sclerosis: update on current trials and perspectives.” Curr Opin Neurol 29 (2016), pp. 199-204.

$[4]$

D. M. Wingerchuk and J. L. Carter. "Multiple sclerosis: current and emerging disease-modifying therapies and treatment strategies." Mayo Clin Proc 89 (2014), pp. $225-240$.

L. J. Balcer, D. H. Miller, S. C. Reingold, et al. "Vision and vision-related outcome measures in multiple sclerosis.” Brain 138 (2014), pp. $11-27$.

$[6]$

A. Petzold, M. P. Wattjes, F. Costello, et al. "The investigation of acute optic neuritis: a review and proposed protocol.” Nat Rev Neurol 10 (2014), pp. 447-458. 
S. R. Hinson, V. A. Lennon, and S. J. Pittock. “Autoimmune AQP4 channelopathies and neuromyelitis optica spectrum disorders.” Handb Clin Neurol 133 (2016), pp. $377-403$.

[9]

A. Petzold, S. Wong, and G. T. Plant. “Autoimmunity in visual loss.” Handb Clin Neurol 133 (2016), pp. 353-376.

$[10]$

B. A. Raphael, K. M. Galetta, D. A. Jacobs, et al. "Validation and test characteristics of a 10-item neuro-ophthalmic supplement to the NEI-VFQ-25." Am J Ophthalmol 142 (2006), pp. 1026-1035.

[11]

C. L. Fraser, I. Davagnanam, M. Radon, et al. "The time course and phenotype of Uhthoff phenomenon following optic neuritis.” Mult Scler 18 (2012), pp. 10421044 .

[12]

L. Leocani, M. A. Rocca, and G. Comi. "MRI and neurophysiological measures to predict course, disability and treatment response in multiple sclerosis." Curr Opin Neurol 29 (2016), pp. 243-253.

$[13]$

J. V. Odom, M. Bach, M. Brigell, et al. "ISCEV standard for clinical visual evoked potentials: (2016 update).” Doc Ophthalmol 133 (2016), pp. 1-9.

[14]

G. E. Holder. "Pattern electroretinography (PERG) and an integrated approach to visual pathway diagnosis.” Prog Retin Eye Res 20 (2001), pp. 531-561.

$[15]$

C. L. Fraser and G. E. Holder. "Electroretinogram findings in unilateral optic neuritis.” Doc Ophthalmol 123 (2011), pp. 173-178.

$[16]$

Z. S. Schnurman, T. C. Frohman, S. C. Beh, et al. "Retinal architecture and mfERG: Optic nerve head component response characteristics in MS.” Neurology 82 (2014), pp. 1888-1896.

[17]

A. T. Toosy, D. F. Mason, and D. H. Miller. “Optic neuritis.” Lancet Neurol 13 (2014), pp. 83-99.

$[18]$

S. Schippling, L. Balk, F. Costello, et al. "Quality control for retinal OCT in multiple sclerosis: validation of the OSCAR-IB criteria." Mult Scler 21 (2015), pp. 163170 .

[19]

P. Tewarie, L. Balk, F. Costello, et al. "The OSCAR-IB Consensus Criteria for Retinal OCT Quality Assessment.” PLoS One 7 (2012), e34823.

$[20]$

A. Cruz-Herranz, L. J. Balk, T. Oberwahrenbrock, et al. "The APOSTEL recommendations for reporting quantitative optical coherence tomography studies."

Neurology 86 (2016), pp. 2303-2309.

[21]

A. Petzold and G. T. Plant. "Diagnosis and classification of autoimmune optic neuropathy." Autoimmun Rev 13 (2014), pp. 539-545.

[22]

I. Gabilondo, E. H. Martı nez-Lapiscina, E. Fraga-Pumar, et al. “Dynamics of retinal injury after acute optic neuritis.” Annals of Neurology 77 (2015), pp. 517-528.

$[23]$

A. Petzold, J. F. de Boer, S. Schippling, et al. "Optical coherence tomography in multiple sclerosis: a systematic review and meta-analysis.” Lancet Neurol 9 (2010), pp. 921-932.

[24]

L. J. Balk, A. Cruz-Herranz, P. Albrecht, et al. "Timing of retinal neuronal and axonal loss in MS: a longitudinal OCT study.” J Neurol 263 (2016), pp. 1323-1331.

$[25]$

E. C. Graham, Y. You, C. Yiannikas, et al. "Progressive Loss of Retinal Ganglion Cells and Axons in Nonoptic Neuritis Eyes in Multiple Sclerosis: A Longitudinal Optical Coherence Tomography Study.” Invest Ophthalmol Vis Sci 57 (2016), pp. 2311-2317.

$[26]$

B. Knier, P. Schmidt, L. Aly, et al. "Retinal inner nuclear layer volume reflects response to immunotherapy in multiple sclerosis.” Brain (2016).

[27]

A. Petzold. "Retinal glymphatic system: an explanation for transient retinal layer volume changes?" Brain (2016).

$[28]$

W. Zhao, S. Stutzman, O. DaiWai, et al. "Inter-device reliability of the NPi-100 pupillometer.” J Clin Neurosci (2016). 
À. Rovira, M. P. Wattjes, M. Tintoré, et al. "Evidence-based guidelines: MAGNIMS consensus guidelines on the use of MRI in multiple sclerosis-clinical implementation in the diagnostic process." Nat Rev Neurol 11 (2015), pp. 471-482.

[30]

M. Wattjes, M. Steenwijk, and M. Stangel. "MRI in the Diagnosis and Monitoring of Multiple Sclerosis: An Update.” Clin Neuroradiol 25 Suppl 2 (2015), pp. 157165.

[31]

R. S. Samson, M. Kolappan, D. L. Thomas, et al. "Development of a high-resolution fat and CSF-suppressed optic nerve DTI protocol at 3T: application in multiple sclerosis." Funct Neurol 28 (2013), pp. 93-100.

[32]

D. A. Paul, E. Gaffin-Cahn, E. B. Hintz, et al. "White matter changes linked to visual recovery after nerve decompression.” Sci Transl Med 6 (2014), $266 \mathrm{ra173.}$

[33]

V. Biberacher, P. Schmidt, A. Keshavan, et al. "Intra- and interscanner variability of magnetic resonance imaging based volumetry in multiple sclerosis." Neuroimage (2016).

[34]

J. B. McKee, J. Elston, N. Evangelou, et al. "Amiloride Clinical Trial In Optic Neuritis (ACTION) protocol: a randomised, double blind, placebo controlled trial." BMJ Open 5 (2015), e009200.

[35]

R. C. Sergott, J. L. Bennett, P. Rieckmann, et al. "ATON: results from a Phase II randomized trial of the B-cell-targeting agent atacicept in patients with optic neuritis.” J Neurol Sci 351 (2015), pp. 174-178.

[36]

J. M. Burton, M. Eliasziw, J. Trufyn, et al. “A prospective cohort study of vitamin D in optic neuritis recovery.” Mult Scler (2016).

[37]

F. Mei, S. P. J. Fancy, Y.-A. A. Shen, et al. "Micropillar arrays as a high-throughput screening platform for therapeutics in multiple sclerosis.” Nat Med 20 (2014), pp. 954-960.

[38]

J. D. Trobe, P. C. Sieving, K. E. Guire, et al. "The impact of the optic neuritis treatment trial on the practices of ophthalmologists and neurologists." Ophthalmology 106 (1999), pp. 2047-2053.

[39]

E. Le Page, D. Veillard, D. A. Laplaud, et al. "Oral versus intravenous high-dose methylprednisolone for treatment of relapses in patients with multiple sclerosis (COPOUSEP): a randomised, controlled, double-blind, non-inferiority trial.” Lancet 386 (2015), pp. 974-981.

[40]

R. L. Gal, S. S. Vedula, and R. Beck. "Corticosteroids for treating optic neuritis.” Cochrane Database Syst Rev (2015), p. CD001430.

[41]

E. Osinga, B. van Oosten, W. de Vries-Knoppert, et al. "Hyperacute steroid therapy in patients with recurrent optic neuritis." MULTIPLE SCLEROSIS JOURNAL.

Vol. 21. SAGE PUBLICATIONS LTD 1 OLIVERS YARD, 55 CITY ROAD, LONDON EC1Y 1SP, ENGLAND. 2015, pp. 627-628.

[42]

G. T. Plant, N. A. Sibtain, and D. Thomas. "Hyperacute corticosteroid treatment of optic neuritis at the onset of pain may prevent visual loss: a case series." Mult Scler Int 2011 (2011), p. 815068.

[43]

K.-W. Sühs, K. Hein, M. B. Sättler, et al. “A randomized, double-blind, phase 2 study of erythropoietin in optic neuritis.” Ann Neurol 72 (2012), pp. 199-210.

[44]

R. Diem, F. Molnar, F. Beisse, et al. "Treatment of optic neuritis with erythropoietin (TONE): a randomised, double-blind, placebo-controlled trial-study protocol." BMJ Open 6 (2016), e010956.

[45]

R. Raftopoulos, S. J. Hickman, A. Toosy, et al. "Phenytoin for neuroprotection in patients with acute optic neuritis: a randomised, placebo-controlled, phase 2 trial." Lancet Neurol 15 (2016), pp. 259-269.

[46]

G. Pihl-Jensen, A. Tsakiri, and J. L. Frederiksen. "Statin treatment in multiple sclerosis: a systematic review and meta-analysis.” CNS Drugs 29 (2015), pp. 277-291.

[47]

A. Tsakiri, K. Kallenbach, D. FuglÃ, et al. “Simvastatin improves final visual outcome in acute optic neuritis: a randomized study.” Mult Scler 18 (2012), pp. 72-81. 


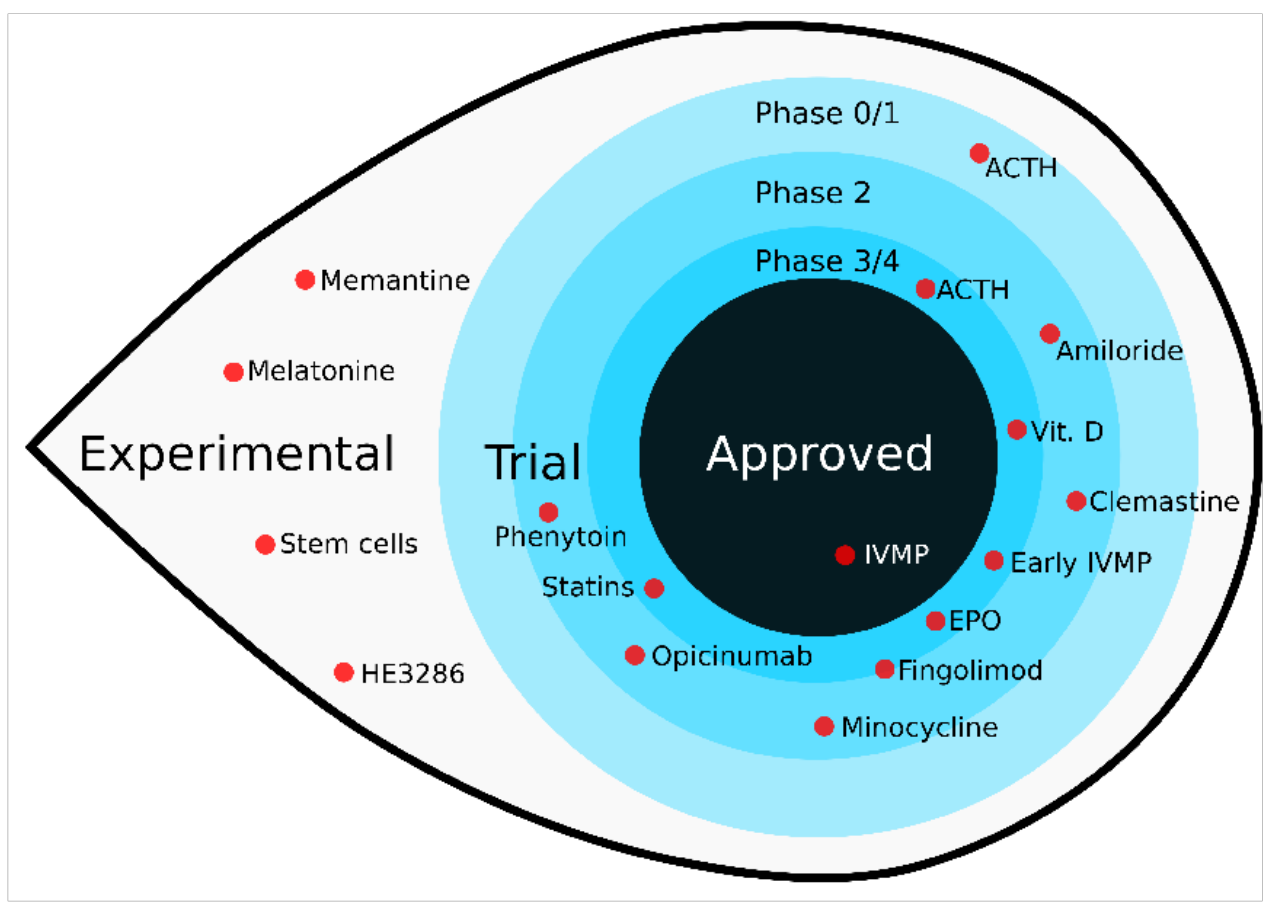

Figure 1: Neuroprotective treatment trials and strategies in MSON. 